\title{
Knabenlernzeiten - Mädchenlernzeiten: gleich, gleicher, ungleich? Genderspezifische Differenzierungen schulischen Wissens
}

\section{Anja Giudici und Karin Manz}

Lange definierten Curricula die Lernzeiten von Mädchen und Knaben unterschiedlich. Erklärt wurde dies bisher mit dem dualen Geschlechtermodell: Weil bildungspolitische Akteure davon ausgingen, Männer und Frauen hätten je unterschiedliche, schulisch zu vervollkommnende Anlagen, resultierten nach Geschlecht differenzierte Curricula. In der Langzeitanalyse von Schweizer Lehrplänen ab 1830 finden wir jedoch keine klar duale Struktur des schulischen Wissens. Daraus schliessen wir, dass das zeit-und klassenübergreifende duale Geschlechtermodell nicht ausreicht, um geschlechtsspezifische Differenzierungen zu erklären. Curricula enthalten immer auch regional- und zeitspezifische Antworten aufkonkrete Problemlagen; sie müssen institutionellen, ökonomischen und praktisch-pädagogischen Ansprüchen genügen.

Offizielle Curricula definieren und strukturieren schulisches Wissen, d.h. die zu vermittelnden Inhalte sowie die damit verbundenen Unterrichtsmaterialien und Lernzeiten $^{1}$ - festgehalten in Schulgesetzen, Lektionentafeln und Lehrplänen. ${ }^{2}$ Sie legen fest, welche Elemente "out of the vast universe of possible knowledge» (Apple, 2003, S. 7) der nächsten Generation weitergegeben werden sollen, sowie anhand welcher Fächer und didaktischer Methoden diese zu strukturieren sind. Zwar entsprechen offizielle Curricula nie dem, was in Schulen tatsächlich gelehrt oder gelernt wird. ${ }^{3}$ Sie nehmen jedoch eine fundamentale Doppelfunktion in Bezug auf das schulische Wissen ein (Westbury et al., 2016). Einerseits legen Curricula zentrale Rahmenbedingungen für die Praxis fest, indem sie bestimmen, wie das schulische Wissen organisiert wird und welche Gruppen von Lernenden Zugang zu welchem Wissen bekommen sollen. Andererseits stellen insbesondere Lehrpläne eine für Öffentlichkeit und Politik zugängliche, relativ konzise Definition dessen dar, was Schule tut und zu welchem Ziel sie das tut. Damit tragen Curricula massgeblich zur Legitimation von Schule als Institution bei.

Seit der Gründung des modernen Schulwesens waren Lernzeiten und Lerninhalte nicht immer gleich für Mädchen und Knaben. Doch erst im Nachgang der zweiten Frauenbewegung kam in der Schweiz die Auffassung, geschlechts- 
spezifisch differenzierte Curricula implizierten eine Diskriminierung, im politischen Mainstream an. Ab den 1970er-Jahren forderte auch die Schweizerische Konferenz der kantonalen Erziehungsdirektoren (EDK) von den Kantonen, Mädchen- und Knabencurricula zu harmonisieren; 1981 hielt sie explizit fest: «Mädchen und Knaben ist während der obligatorischen Schulzeit dieselbe Ausbildung anzubieten» (EDK, 1981, Punkt 1; vgl. dazu auch Crotti, 2006). Mit dem im selben Jahr angenommenen Gleichstellungsartikel (Art. 4, Abs. 2 BV 1999) konnten entsprechende Abweichungen nunmehr eingeklagt werden. Doch es bedurfte bis zur tatsächlichen Vereinheitlichung der Curricula langwieriger Reformprozesse, einer erneuten EDK-Empfehlung (EDK, 1993) sowie einer Reihe Beschwerden engagierter Eltern, wovon eine zu einem Bundesgerichtsurteil führte, das untersagte, «in der Ausbildung zwischen Mädchen und Knaben Unterscheidungen zu treffen, die nicht biologisch und funktionell begründet sind» (Bundesgericht, 1986, S. 172). Erst in den 1990er-Jahren entstanden in allen Kantonen formal geschlechtsneutrale Lehrpläne, die Lektionentafeln und Lernzeiten nicht mehr nach Geschlecht differenzierten.

Dieser Schritt beendete allerdings keineswegs die Diskussion um Curricula und Fächer als "gegenderte [engl. engendered] Domänen» (Willems, 2007, S. 81). Spätestens seit den internationalen Leistungstests und dem Ausrufen der «boy crisis» (Mead, 2006, S. 3) stehen die Wirkung von Curricula auf geschlechtsspezifische (Un-)Gleichheit sowie der Nutzen und Schaden der Koedukation wieder im Fokus der öffentlichen und fachlichen Debatte (vgl. Sullivan, Joshi \& Leonard, 2010). Diesmal unter umgekehrten Vorzeichen, wie die gegenwärtige Diskussion zur schulischen Diskriminierung der Knaben durch die angebliche Sprachlastigkeit der Lehrpläne und Orientierung der Curricula an Interessen und Anlagen der Mädchen zeigt.

Unser Beitrag leistet eine Historisierung der Debatte und fragt nach ihren theoretischen Hintergründen. Wir fragen, ob und wie Curricula in der Geschichte der Volksschule Lernzeiten und schulisches Wissen nach Geschlecht differenzierten, und evaluieren potentielle Erklärungen für dieses Phänomen. Wir analysieren ausgehend von den Ergebnissen des SNF-Sinergia-Projektes «Die Transformation schulischen Wissens seit 1830»4 Schulgesetze und Lehrpläne auf ihre geschlechtsspezifischen Differenzierungen hin und stellen unsere daraus abgeleitete Systematik vor. Darüber hinaus diskutieren wir, ob das bürgerlich-duale Geschlechtermodell als Erklärungsansatz für die geschlechtsspezifische Prägung von schulischen Curricula taugt, und entwickeln mögliche Alternativen. Unsere Analysen zeigen, dass das klassische Geschlechtermodell durchaus das Denken relevanter Akteure in der Lehrplanarbeit strukturierte. Allerdings bieten die in der Zeit- und Raumperspektive äusserst stabilen Ideen und geteilten Normen zur Anlage der Geschlechter keine ausreichenden Erklärungen für die im diachronen und synchronen Vergleich äusserst heterogene Ordnung curricularer Differenzierungen. Weitaus aufschlussreicher sind ökonomische Aspekte und soziale Zuschreibungen zu zukünftigen Berufs- und Lebensentwürfen von Mädchen und Knaben. 


\section{Das bürgerliche, duale Geschlechtermodell ...}

Gemäss der Geschlechterforschung bildete sich spätestens seit der Moderne ein duales Geschlechtermodell mit je unterschiedlichen Zuschreibungen und Charakterisierungen für Frauen und Männer aus. Geprägt und für die Bildungsforschung fruchtbar gemacht wurde dieses Konzept in Grundlagewerken wie Hausens Polarisierung der "Geschlechtscharaktere» (1976) oder Smiths Ladies of the leisure class (1981). Beide Werke legen dar, wie mit dem Aufstieg des Bürgertums im 18. Jahrhundert spezifisch bürgerliche Vorstellungen über die partikuläre Aufgaben- und Rollenteilung zwischen Mann und Frau zur schichtunabhängigen, allgemein anerkannten Norm wurden. ${ }^{5}$ Das duale Geschlechtermodell setzte sich gegenüber alternativen Modellen durch und blieb bis in die zweite Hälfte des 20. Jahrhunderts relativ stabil. In Wissenschaft und Kultur wurden Männern und Frauen Geschlechtercharaktere zugeteilt und diese Zuschreibungen mit deren grundsätzlich unterschiedlichen, «natürlichen» Anlagen legitimiert. Frauen galten demnach als passiv, gefühlvoll, bescheiden und nach innen gewandt; Männer hingegen seien aktiv, vernünftig, tapfer und für das öffentliche Leben bestimmt.

Die historische Bildungsforschung hat in Bezug auf Geschlechterfragen insbesondere die höhere Mädchenbildung fokussiert und wiederholt auf die Wirkungskraft der dualen Norm auf die geschlechtsspezifische Strukturierung von Curricula hingewiesen. Insbesondere die Dominanz bürgerlicher bzw. kirchlicher Akteure und deren "gendered attitudes", so Albisetti, Goodman und Rogers (2010, S. 4), erklärt die Persistenz dieser Norm in diesem Schulbereich. Dies führte gemäss Literatur zu drei Effekten.

Erstens löste sich das duale Geschlechtermodell allmählich von seiner bürgerlichen Prägung und wurde zu einer zeit-, schicht- und kontextunabhängigen Norm (Hausen, 1976; Mayer, 1999; Smith, 1981). Gemäss vergleichender Forschung strukturierte dieses Geschlechtermodell über den europäischen Raum hinweg und bis in die zweite Hälfte des 20. Jahrhunderts die Curricula höherer Sekundarschulen massgeblich (Albisetti, Goodmann \& Rogers, 2010).

Zweitens produzierte das duale Modell Affinitäten zwischen bestimmten Wissensbeständen und dem einen oder dem anderen Geschlecht. Damit galten auch schulische Fächer entweder als eher «männlich» oder «weiblich» konnotiert. Auch Bourdieu (2002, S. 119) stellt in seinem Exkurs zur Geschlechterforschung eine solche Zweiteilung fest, zwischen männlichen «disciplines dures» wie die Physik und weiblichen "disciplines molles» wie die Sozial- und Geisteswissenschaften. Andere Forschende kommen auf ähnliche Schlüsse bezüglich geschlechtsspezifischer Prägung bestimmter Fächer und Wissensbestände (Lojewski, 2007; Tolley, 2003; Willems, 2007). Diese Zuschreibungen führten zur stärkeren Gewichtung "harter» Fächer in Knaben- und «weicher» Fächer in Mädchensekundarschulcurricula. 
Drittens führte das duale Geschlechtermodell $\mathrm{zu}$ differenten Bildungskonzepten. Mayer (1999) und Schmid (1986) stellen fest, dass die den klassischen Bildungstheorien zugrundeliegende Unterscheidung zwischen zweckfreier Allgemeinbildung und nützlicher Berufsbildung nur die Bildung der Knaben betraf. Ausgehend vom dualen Geschlechtermodell sahen Pädagogen die Bestimmung der Frau hingegen im dreifachen Lebensberuf der Frau als Mutter, Gattin und Hausfrau, womit auch ihr Konzept der Mädchenbildung Beruf und (Allgemein-) Bildung als Einheit sah. Dieses Konzept bezog sich gemäss Mayer (1999) zunächst auf die Bildung bürgerlicher Frauen und wurde dann zur schichtunabhängigen Norm, während es gleichzeitig ausgeweitet wurde, um sowohl die Vorbereitung auf Familien- und/oder Erwerbsarbeit zu integrieren. Als Konsequenz zielten Sekundarschulen mehrheitlich auf die Allgemeinbildung «öffentlicher Männer» (Albisetti, Goodmann \& Rodgers, 2010, S. 3), während sich die Vorbereitung auf die "häusliche Mütterlichkeit» (S. 5) als roter Faden durch die europäische Mädchensekundarbildung zieht.

\section{... bestimmt das Denken und die Lehrplanarbeit.}

Für die Schweiz übertragen zwei Studien die These eines prägenden Einflusses des dualen Geschlechtermodells auf die Volksschule. Mantovani Vögeli (1994) deutet die gesamte Entwicklung der Schweizer Mädchenbeschulung vor dem Hintergrund "polare[r] Zuschreibungen von weiblichen und männlichen Wesensmerkmalen», die «in die Köpfe von Frauen und Männern» (S. 30) eingeprägt waren. Kellerhals (2010) erklärt die zunehmende Geschlechterdifferenzierung der bernischen Lehrpläne mit dem Einfluss bürgerlicher Kreise und hält fest: «Am Ende des 19. Jahrhunderts entsprach die inhaltliche Ausrichtung der Volksschule [...] von vom Hauptstrom angestrebten ideologischen Ansprüchen einer arbeitsteiligen bürgerlichen Gesellschaft» (S. 241).

In der Tat weisen die Äusserungen führender Akteure der Schweizer Curriculumpolitik des 19. und frühen 20. Jahrhunderts solche polaren Zuschreibungen auf. Für den liberalen Luzerner Seminardirektor und Lehrplanautor Dula (1873) «sind die intellektuelle[n] Fähigkeiten der beiden Geschlechter verschieden». Der Knabe «hat Freude an dem, was gross und stark ist; seine Intelligenz entwickelt sich nur langsam, aber sein Denken wird später umso ernster und tiefer. Das Mädchen gibt gewöhnlich dem Schönen und Zarten den Vorzug»(S. 5). Auch Schulinspektor Wagner (1870) sah bei Mädchen eine schwächere «Denkkraft [...], weil die Organisation des Gehirnes zarter und mehr zur Fettbildung neigt» (S. 11); und Schulinspektor Kettiger (1854) stellte unmissverständlich fest: «Des Mannes Haus ist die Welt und des Weibes Welt ist das Haus» (S. 39). Die Basler Mädchenschulrektorin Hauri (1965) konstatierte noch 1965, angesichts der technischen Fortschritte müssten Lehrpläne «im Mädchen und damit in der künftigen Gattin und Mutter das Gemütsvolle, Mütterliche und Bewahrende fördern» (S. 185). 
Inwieweit wirken sich solche Geschlechterbilder auf Lehrpläne und Lernzeiten aus? - Wäre die These eines direkten Einflusses des dualen Geschlechtermodells auf Volksschulcurricula übertragbar, dann müssten sich die Stabilität und Einheitlichkeit, die diese Norm charakterisieren, auch in den historischen Daten widerspiegeln. Spezifisch müssten die drei zuvor für die höhere Bildung diskutierten Implikationen auch für die Volksschule beobachtbar werden.

Gesetzt den Fall, die oben formulierte Übertragbarkeit würde zutreffen, müssten wir erstens aus einer synchron-vergleichenden Perspektive zwischen verschiedenen kantonalen Lehrplänen sowie aus einer diachron-vergleichenden Perspektive über die Zeit hinweg relativ einheitliche und stabile Differenzierungen in den Lehrplänen vorfinden, unabhängig des konfessionellen, regionalen oder wirtschaftlichen Kontextes. Zweitens müsste je ein relativ klar umrissener Korpus von spezifischen Mädchen- und Knabenfächern vorhanden sein. Drittens müsste in Knabenlehrplänen der Primar- und Sekundarschulen ein eher allgemeinbildend und nicht an die zukünftigen Berufsperspektiven gerichteter Fächerkanon ersichtlich werden, währenddessen die Mädchenlehrpläne allgemein- und berufsbildende Fächer verbinden würden. - Ist das empirisch nachweisbar?

Um dies zu prüfen, greifen wir auf die von uns im Teilprojekt $\mathrm{C}$ «Die Lehrpläne in der deutschsprachigen Schweiz seit 1830 - Inhalte und Konstruktionsprinzipien schulischen Wissens im Wandel» durchgeführten Analysen zurück (vgl. Fussnote 4). Wir kontrastieren die oben formulierten Implikationen einerseits mit einer vergleichenden Analyse quantitativer und inhaltlicher Angaben zur Strukturierung der Schulzeit für Mädchen und Knaben in den Curriculumdokumenten aus einer diachronischen Perspektive über die Zeit sowie aus einer synchronen Perspektive zwischen den Kantonen. ${ }^{6}$ Andererseits analysieren wir inhaltlich die Aussagen der in den Curriculumarbeit involvierten Akteure, um ihre Handlungslogik und die Gründe hinter ihren Entscheiden zu rekonstruieren.

\section{Heterogene Lehrpläne}

Gemäss dem Postulat Bildung für alle und der Verstaatlichung der Schulsysteme führten die meisten Kantone in den 1830er-Jahren für die Primarschule ein einheitliches Programm für beide Geschlechter ein. Wir finden in dieser Zeit in der Regel für alle Schülerinnen und Schüler einen einzigen Lehrplan mit einheitlicher Lektionentafel. Unter Ägide des radikalen Regimes lernten bspw. in der freiburgischen Primarschule um 1850 Mädchen und Knaben zunächst dasselbe - wie die entsprechende Spalte in Tabelle 1 zeigt. Insbesondere in städtischen Gebieten wie Zürich oder Basel hingegen bestanden zu diesem Zeitpunkt ab Primarschulstufe bereits parallele Schulsysteme oder Differenzierungen, in denen Mädchen und Knaben je spezifisches schulisches Wissen vermittelt 
wurde. Diese frühen städtischen Lehrpläne verpflichten bereits Primarschülerinnen zu Handarbeitslektionen. Der stadtzürcherische Lehrplan von 1833 schreibt ab dem ersten Schuljahr vier Lektionen Handarbeit vor, ab dem zweiten sechs und ab dem fünften acht. Im Rahmen der Kantonalisierung der Curricula (Giudici et al., in Druck) setzte sich dieses Modell durch: Spätestens in der zweiten Hälfte des 19. Jahrhunderts wurden in der Schweiz alle Mädchen zum Besuch des weiblichen Handarbeitsunterrichts verpflichtet - lange das geschlechtsspezifische Fach par excellence. Damit verlängerte sich jedoch ihre Schulwoche gegenüber derjenigen der Knaben um vier bis sechs Mehrstunden, wie im Freiburger Lehrplan von 1876 ersichtlich.

Tabelle 1. Lektionentafel Primarschule / École primaire FR, 4. Schuljahr

\begin{tabular}{|c|c|c|c|c|c|c|}
\hline Fach/Lehrplan & 1850 & 1876 & 1886 & 1889 & 1893 & 1974 \\
\hline Religion & 1.5 & 1.75 & 2.5 & 5 & 5 & 2 \\
\hline Schulsprache & 7.75 & 9 & $\begin{array}{l}9 \mathrm{~m} \\
7.5 \mathrm{w}\end{array}$ & $\begin{array}{c}11 \mathrm{~m} \\
10.5 \mathrm{w}\end{array}$ & $\begin{array}{l}9 \mathrm{~m} \\
8 \mathrm{w}\end{array}$ & $\begin{array}{l}9 \mathrm{~m} \\
8 \mathrm{w}\end{array}$ \\
\hline Realien & 0.75 & 1.5 & $\begin{array}{l}6 \mathrm{~m} \\
5 \mathrm{w}\end{array}$ & $\begin{array}{c}2.5 \mathrm{~m} \\
2 \mathrm{w}\end{array}$ & $\begin{array}{l}3.5 \mathrm{~m} \\
2.5 \mathrm{w}\end{array}$ & 4 \\
\hline Mathematik & 5.25 & 4.75 & $\begin{array}{c}5.5 \mathrm{~m} \\
4 \mathrm{w}\end{array}$ & 5 & $\begin{array}{l}5 \mathrm{~m} \\
4 \mathrm{w}\end{array}$ & 4 \\
\hline Zeichnen & 0.75 & 0 & 0 & $1 \mathrm{~m}$ & 0 & \multirow{5}{*}{$\begin{array}{l}5 \mathrm{~m} \\
6 \mathrm{w}\end{array}$} \\
\hline Schreiben & 1.5 & 2.5 & 1 & 1 & 1 & \\
\hline Singen & 1.25 & 0.5 & 1 & 1 & 1 & \\
\hline Handarbeit/Werken & 0 & $4.5 \mathrm{w}$ & $3 \mathrm{w}$ & \multirow{2}{*}{$3 w$} & \multirow{2}{*}{$5.5 \mathrm{w}$} & \\
\hline Haushaltskunde & & & $1 \mathrm{w}$ & & & \\
\hline Turnen & 0 & 0 & 0 & 0 & $\begin{array}{c}2 \mathrm{~m} \\
0.5 \mathrm{w}\end{array}$ & 3 \\
\hline Summe & 18.75 & $\begin{array}{c}20 \mathrm{~m} \\
24.5 \mathrm{w}\end{array}$ & 25 & $\begin{array}{l}26.5 \mathrm{~m} \\
27.5 \mathrm{w}\end{array}$ & 27.5 & 30 \\
\hline
\end{tabular}

Legende: Die Zahlen bezeichnen die Anzahl Lektionen, die Primarschülerinnen und -schüler des 4. Schuljahres im Jahresdurchschnitt pro Woche in den verschiedenen Fachbereichen absolvieren sollen, gemäss verabschiedetem Schulgesetz oder Lehrplan (Jahr in der ersten Zeile angegeben). $\mathrm{m}=$ Knaben; $\mathrm{w}=$ Mädchen; SJ = Schuljahr. Es sind die Fachbereiche eingefärbt, die für Mädchen und Knaben unterschiedliche Lektionendotationen vorsehen.

Mit der Zeit wurde eine Mehrbelastung der Mädchen von den zuständigen Behörden als «unstatthaft» (Lehrplan [LP] Prim BE 1897, S. 12) kritisiert. Die «vermehrte Inanspruchnahme der Mädchen [sei] schon deshalb nicht ohne Bedeutung, weil Mädchen in der Regel durch das Haus in stärkerem Masse in Anspruch genommen werden» (LP Volksschule ZH 1905, S. 13). In gewissen 
Kantonen begannen Lehrpläne und Schulgesetze Kompensationsfächer zu definieren, in anderen Fällen wurde die Auswahl den Lehrpersonen und lokalen Behörden überlassen. Obwohl Curricula mit unterschiedlich langen Wochen teilweise bis in die 1960er-Jahre weiterbestanden, zeugen die Lektionentafeln ab dem späten 19. Jahrhundert vom Bestreben der Entscheidungsträger, die Lernzeiten in der Summe anzugleichen. ${ }^{7}$ In Freiburg beispielsweise werden ab 1886 die vier bis sechs wöchentlichen Lektionen Handarbeit für Mädchen durch Abstriche in anderen Fächern (fast) gänzlich kompensiert (vgl. Tabelle 1). Dies bedingte, dass die Lektionenzahl in bestimmten Fächern für Mädchen reduziert wurde, wodurch das schulische Wissen eine stärkere genderspezifische Ausprägung erhielt.

\section{Definierte «Mädchen- und Knabenfächer»?}

Im Grundsatz schienen sich Pädagogen und Behörden einig: Mädchen sollten als Ausgleich zum Besuch der Handarbeit in den Fachbereichen dispensiert werden, die "weniger in den Bildungskreis der Mädchen gehören» (Volksschulgesetz [VSG] ZH 1859, Art. 111), bzw. der Handarbeitsunterricht sollte auf die Zeit gelegt werden, «in welcher den Knaben der für ihre besondere Ausbildung bestimmte Unterricht ertheilt wird»(VSG AG 1865, Art. 51). Der interkantonale Vergleich zeigt jedoch, dass in fast allen Fachbereichen zeitliche Kürzungen für Mädchen möglich waren: Sprachen, Realien, Mathematik, Schreiben, Singen. Manchmal waren Teile des Schreib-, Sprach- oder Mathematikunterrichts (Buchhaltung, Geometrie, Algebra) für Mädchen fakultativ, manchmal waren sie ausschliesslich Knaben vorbehalten, manchmal für alle obligatorisch - die Auswahl scheint teilweise gar widersprüchlich. So schrieben die Luzerner Volksschullehrpläne der 1890er-Jahre vor, dass in der Zeit, in der Mädchen die Arbeitsschule besuchten, Jungen in Turnen, Geometrie, Verfassungskunde und Sprache unterrichtet werden sollten (LP Prim LU 1890; LP Sek LU 1895). Der im selben Zeitraum erlassene Tessiner Lehrplan führte hingegen das Obligatorium der Verfassungskunde für beide Geschlechter ein, "perché nessuno ha il diritto di crescere nella ignoranza delle cose risguardanti la patria e i diritti ed i doveri del cittadino" (LP Elementari TI 1894, S. 16). Demgegenüber verbot der Zürcher Sekundarschullehrplan explizit, die Mädchen vom Turnunterricht zu dispensieren, «denn dieses Fach hat für die Mädchen nicht minder Bedeutung als für die Knaben» (LP Volksschule ZH 1905, S. 14).

Auch im Zeitvergleich zeigt sich am Beispiel Kanton Basel-Stadt (vgl. Tabelle 2), dass bis in die 1960er- und 1970er-Jahre eigentlich alle Fächer von Differenzierungen betroffen sein konnten. Wie in Basel-Stadt erhielten Mädchen auch in anderen Kantonen zunächst insbesondere in Fachbereichen mit hoher Lektionendotation weniger Lektionen, danach verschoben sich die Differenzierungen. Auffallend ist, dass in den Fachbereichen Mathematik, (Technisches) Zeichnen oder Turnen, die von Erziehungsexperten und der breiteren Gesellschaft ab dem 19. Jahrhundert als eher «männlich» konnotiert wahrgenommen werden 
(Lojewski, 2011; Tolley, 2003; Willems, 2007), für Mädchen und Knaben explizit gleich viel Lektionen vorgesehen waren.

Die empirischen geschlechtsspezifischen Differenzierungen lassen sich daher eher mit dem Prinzip der (uneinheitlichen) Kompensation charakterisieren, als mit der Schaffung eines je kohärenten Programms zur Erziehung der Geschlechter: Weder lassen sich, mit Ausnahme von Handarbeit/Hauswirtschaft sowie Werken klare «Mädchen- oder Knabenfächer» erkennen, noch sind die Differenzierungen im synchronen oder diachronen Vergleich so stabil, als dass sie mit ausschliesslichem Bezug auf das duale Geschlechtermodell erklärt werden könnten.

Tabelle 2. Lektionentafeln Primarschule und Sekundarschule (obere Primarschule) Basel-Stadt

\begin{tabular}{|c|c|c|c|c|c|c|c|c|c|c|}
\hline \multirow{2}{*}{$\begin{array}{l}\text { Fach/ } \\
\text { Lehrplan }\end{array}$} & \multicolumn{6}{|c|}{ Primarschule, 1. - 4. Schuljahr } & \multicolumn{4}{|c|}{ Sekundarschule 5. - 8. Schuljahr } \\
\hline & $1871 / 72$ & $1884 / 90$ & 1900 & 1933 & 1969 & 1991 & $1883 / 84$ & $892 / 1904$ & $1931 / 32$ & 1976 \\
\hline Religion & $\begin{array}{l}3 \mathrm{~m} \\
2 \mathrm{w}\end{array}$ & $\begin{array}{c}2.5 \mathrm{~m} \\
2 \mathrm{w}\end{array}$ & $\begin{array}{l}2.5 \mathrm{~m} \\
2 \mathrm{w}\end{array}$ & & & 1.75 & 1 & 1 & & \\
\hline Sprachen & $\begin{array}{c}10.7 \mathrm{~m} \\
8 \mathrm{w}\end{array}$ & $\begin{array}{l}9.3 \mathrm{~m} \\
7.8 \mathrm{w}\end{array}$ & $\begin{array}{l}9.3 \mathrm{~m} \\
7.8 \mathrm{w}\end{array}$ & $\begin{array}{l}9.5 \mathrm{~m} \\
8.3 \mathrm{w}\end{array}$ & 7.8 & \multirow{2}{*}{6.8} & $\begin{array}{l}11 \mathrm{~m} \\
8 \mathrm{w}\end{array}$ & $\begin{array}{c}11 \mathrm{~m} \\
8 \mathrm{w}\end{array}$ & 6 & 8 \\
\hline Realien & & 0.5 & 0.5 & 0.5 & & & $\begin{array}{c}5.3 \mathrm{~m} \\
5 \mathrm{w}\end{array}$ & $\begin{array}{c}5.3 \mathrm{~m} \\
5 \mathrm{w}\end{array}$ & $\begin{array}{l}6.3 \mathrm{~m} \\
3.8 \mathrm{w} \\
\end{array}$ & 5.5 \\
\hline $\begin{array}{l}\text { Mathe- } \\
\text { matik }\end{array}$ & $\begin{array}{l}5.7 \mathrm{~m} \\
3.7 \mathrm{w}\end{array}$ & $\begin{array}{l}4.8 \mathrm{~m} \\
4.5 \mathrm{w}\end{array}$ & $\begin{array}{l}5 \mathrm{~m} \\
4.3 \mathrm{w}\end{array}$ & 4.5 & 4.5 & 4.8 & 4 & 4 & $\begin{array}{l}6 \mathrm{~m} \\
5 \mathrm{w}\end{array}$ & 5 \\
\hline Zeichnen & & 0.5 & 0.5 & $\begin{array}{l}0.5 \mathrm{~m} \\
0.3 \mathrm{w}\end{array}$ & 1 & 1.5 & 2 & 2 & 2 & 2 \\
\hline $\begin{array}{l}\text { Geometrie } \\
\text { Tech. } \\
\text { Zeichnen }\end{array}$ & & & & & & & $2 \mathrm{~m}$ & $1 \mathrm{~m}$ & $2 \mathrm{~m}$ & \\
\hline Schreiben & $\begin{array}{c}4.7 \mathrm{~m} \\
2 \mathrm{w}\end{array}$ & 3.5 & 3.5 & 2 & 1 & 1 & $\begin{array}{l}2 \mathrm{~m} \\
1 \mathrm{w}\end{array}$ & 2 & 2 & 1 \\
\hline Singen & $\begin{array}{c}2 \mathrm{~m} \\
1.7 \mathrm{w}\end{array}$ & 1.5 & 1.5 & $\begin{array}{l}1.8 \mathrm{~m} \\
1.5 \mathrm{w}\end{array}$ & 1.3 & 1.5 & $\begin{array}{l}2 \mathrm{~m} \\
1 \mathrm{w}\end{array}$ & $\begin{array}{l}2 \mathrm{~m} \\
1 \mathrm{w}\end{array}$ & $\begin{array}{l}1 \mathrm{~m} \\
2 \mathrm{w}\end{array}$ & 1 \\
\hline $\begin{array}{l}\text { Handarbeit } \\
\text { Werken }\end{array}$ & $6.7 w$ & $4 w$ & $4 w$ & $\begin{array}{c}3.3 \mathrm{w} \\
1 \mathrm{~m}\end{array}$ & $\begin{array}{l}2 \mathrm{~m} \\
3 \mathrm{w}\end{array}$ & 3 & $6 w$ & $5 w$ & $\begin{array}{l}5 \mathrm{w} \\
2 \mathrm{~m} \\
\end{array}$ & 4 \\
\hline $\begin{array}{l}\text { Hauswirt- } \\
\text { schaft }\end{array}$ & & & & & & & & & $2 w$ & 1 \\
\hline Turnen & & $0.5 \mathrm{~m}$ & $1 \mathrm{~m}$ & 3 & 3 & 3 & 2 & 2 & 3 & 3 \\
\hline Anderes & & & & & $1 \mathrm{~m}$ & 2.3 & & & & 2 \\
\hline Summe & $\begin{array}{l}26 \mathrm{~m} \\
24 \mathrm{w}\end{array}$ & $\begin{array}{c}23 \mathrm{~m} \\
24.3 \mathrm{w}\end{array}$ & $\begin{array}{c}23.8 \mathrm{~m} \\
24 \mathrm{w}\end{array}$ & $\begin{array}{l}22.8 \mathrm{~m} \\
23.3 \mathrm{w}\end{array}$ & 21.5 & 25.6 & 30 & 30 & 29 & 31 \\
\hline
\end{tabular}




\section{Allgemeinbildendes versus berufsorientiertes \\ Curriculum?}

Die flächendeckende Verpflichtung der Mädchen zum Besuch der Handarbeit scheint darauf hinzudeuten, dass Mädchencurricula im 19. Jahrhundert tatsächlich Berufs- und Allgemeinbildung integrierten und früher durch eine berufspraktische Orientierung geprägt waren als diejenigen der Knaben. Im Umkehrschluss heisst dies jedoch nicht, dass Volksschulcurricula Knaben nicht mit spezifischem, auf spätere Berufsperspektiven ausgerichtetem Wissen konfrontierten. Bei der Implementierung von Fachbereichen wie (Technisches) Zeichnen, Algebra und Buchhaltung, Werken, Landwirtschaft oder Fremdsprachen orientierten sich Akteure der Lehrplanarbeit an den wirtschaftlichen Bedürfnissen von Region und Familien (Giudici \& Grizelj, 2017; Giudici, 2018). Bereits in den 1840er-Jahren lamentierten humanistische Kreise, dass in der Volksschulbildung Inhalte nicht «um ihrer selbst Willen» eingeführt würden, sondern um «den praktischen Nutzen, den der Knabe dereinst, sei es in diesem oder jenem Berufe, daraus ziehen soll» (Warnende Stimme, 1842, S. 9f.).

Eine neue Dynamik zeichnete sich um 1900 ab und verstärkte sich bis in die 1930er-Jahre. Insbesondere auf der Primaroberstufe wurden einerseits die den Mädchen vorbehaltenen Fächer ausdifferenziert, andererseits wurden neue, stärker berufsspezifisch ausgerichtete - und zunächst fakultative - Unterrichtsgegenstände für Knaben im praktischen, technischen und gestalterischen Bereich als Pendant eingeführt. Damit bildete sich neben einem einheitlichen Curriculum in Schulsprache, Mathematik und anderen Kernbereichen allmählich ein ergänzendes, geschlechtsspezifisches Curriculum aus mit Fächern für Mädchen und Fächern für Knaben. Letzteres sollte beide Geschlechter auf ihre (berufs-) praktischen Aufgaben in der Gesellschaft vorbereiten. So waren bspw. auch die Mehrlektionen Rechnen und Geometrie in den baselstädtischen Lehrplänen (vgl. Tabelle 2) vorgesehen, um den Knaben, «die im täglichen Leben vorkommenden und für den Handwerker wichtigen, einfachen Rechenaufgaben» zu vermitteln bzw. «den Schüler [zu] befähigen, die fürs Handwerk nötigen einfachen geometrischen Konstruktionen zu lösen» (LP Knabensek BS 1931, S. 7, 9). Ähnlich sehen die für Mädchen konzipierten Lehrpläne vor, dass diese dazu befähigt werden für den Alltag «einfache Wäsche- und Kleidungsstücke, wie auch praktische Gegenstände für den Hausgebrauch selbständig zu formen» (LP Mädchensek BS 1931, S. 13), aber auch für das Erwerbsleben Geschäftsbuchhaltung und -korrespondenz erlernen. 


\section{Eine Systematisierung geschlechtsspezifischer Curriculum- und Lernzeitdifferenzierungen}

Wie die bisherigen Ausführungen zeigen, sind geschlechtsspezifische Differenzierungen äusserst heterogen. Wir haben empirie-gestützt ein Modell erarbeitet, das erlaubt, Differenzierungstypen zu unterscheiden und deren Verbreitung in Zeit und Raum einzuordnen (vgl. Tabelle 3).

Die erste Kategorie umfasst geschlechtsneutral verfasste Lehrpläne und Lektionentafeln; diese definieren für einen Zeitraum und Schultyp eine verbindliche Lektioneneinteilung und Inhaltsbeschreibung. Diesen Lehrplantypus finden wir überwiegend in den ersten Jahrzehnten der modernen Volksschule bis Mitte des 19. Jahrhunderts und v.a. auf der Primarstufe in ländlichen Regionen. Geschlechtsneutrale Lehrpläne etablieren sich danach erst wieder Ende der 1980er-, flächendeckend seit den 1990er-Jahren als Standard für alle Volksschulklassen. Diese Reform wird markiert durch die Öffnung der Hauswirtschaft für Knaben und die Integration von Handarbeit und Werken in einen koedukativ ausgerichteten Fachkomplex.

Tabelle 3. Typen der Curriculum- und Lernzeitdifferenzierung nach Geschlecht

\begin{tabular}{|c|c|c|c|}
\hline Differenzierungsart & Knaben & Mädchen & Verbreitung \\
\hline $\begin{array}{l}\text { (1) Gender neutral } \\
\text { curriculum }\end{array}$ & & & $\begin{array}{l}\text { Insbesondere Primarstufe } \\
\text { frühes 19. Jahrhundert; } \\
\text { Curricula nach } 1990\end{array}$ \\
\hline $\begin{array}{l}\text { (2a) Girl-specific } \\
\text { partially engendered } \\
\text { curriculum }\end{array}$ & & & $\begin{array}{l}\text { Vor allem bis Mitte } 19 . \\
\text { Jahrhundert }\end{array}$ \\
\hline $\begin{array}{l}\text { (2b) Girl-specific } \\
\text { compensated partly } \\
\text { engendered curriculum }\end{array}$ & & & $\begin{array}{l}\text { Meistverbreites Modell } \\
\text { ab zweite Hälfte des } 19 . \\
\text { Jahrhunderts }\end{array}$ \\
\hline $\begin{array}{l}\text { (2b) Boy- and girl-spe- } \\
\text { cific partly engendered } \\
\text { curriculum }\end{array}$ & & & $\begin{array}{l}\text { Insbesondere Schultypen } \\
\text { mit Grundansprüchen, } 20 . \\
\text { Jahrhundert }\end{array}$ \\
\hline $\begin{array}{l}\text { (3) Fully engendered } \\
\text { curriculum }\end{array}$ & & & $\begin{array}{l}\text { Vor allem Städte (z.B. } \\
\text { ZH, BS); Curricula versch. } \\
\text { Schultypen mit erweiterten } \\
\text { Ansprüchen auf Sekun- } \\
\text { darstufe I }\end{array}$ \\
\hline
\end{tabular}

Die zweite Kategorie umfasst eine dreischrittige Entwicklung von zusätzlichen, mädchenspezifischen Schulfächern einhergehend mit einer längeren Beschulung für Mädchen (2a) über die Etablierung von vielfältigen Kompensationsmodellen (2b) bis hin zu einem neutralen Kerncurriculum mit je mädchen- und knabenspezifischen Ergänzungen (2c). Letzteren Lehrplantypus finden wir Mitte des 20. Jahrhunderts praktisch in allen Primarschulen, aber vor allem in den 
leistungsanforderungsmässig tieferen Niveaus der Sekundarstufe I. Es entstand eine implizite Zweiteilung der Lehrpläne mit je einem geschlechtsspezifisch-differenzierten, auf die unterschiedlichen Lebenswelten und spätere Tätigkeiten von Knaben und Mädchen ausgerichteten Bereich.

In der dritten Kategorie wird das schulische Wissen vollständig nach Geschlecht der Schülerschaft differenziert. Die seduzierten Lehrpläne sehen für Mädchen und Knaben zwei sowohl in der Zeiteinteilung als auch in den Inhalten unterschiedliche Curricula vor. Ihre Umsetzung verlangt praktisch ein paralleles Mädchen- und Knabenschulwesen mit entsprechend verdoppelter Lehrpersonenausbildung, Lehrmittelproduktion und Infrastruktur. Sie sind relativ selten und finden sich ausschliesslich für städtische Schulverhältnisse mit einer gut ausgebauten Infrastruktur sowie in der höheren Schulbildung. ${ }^{8}$

\section{Vier Erklärungsansätze für Differenzierungen}

Die unsystematische, wechselnde Wahl der Kompensationsfächer, die unscharfe Trennung zwischen Knaben- und Mädchenfächern sowie Allgemein- und Berufsbildung widersprechen der eingangs formulierten These. Es sind einerseits deutliche Hinweise dafür, dass die Akteure der Curriculumarbeit trotz Dominanz und Stabilität des dualen Geschlechtermodells zu unterschiedlichen Schlüssen bezüglich «richtiger» Beschulung von Mädchen und Knaben kamen. Sie zeigen andererseits, dass geschlechtsspezifische Differenzierungen in Volksschulcurricula nicht primär das Ziel verfolgten, zwei parallele, gänzlich der Natur und Bestimmung der Geschlechter konforme Bildungsprogramme zu etablieren. Zum Abschluss skizzieren wir vier alternative Erklärungsansätze für die in unseren Daten vorgefundenen differenzierten Differenzierungen.

\section{Volksschule für alle}

Volksschulcurricula scheinen weit weniger ausgeprägte Differenzierungen aufzuweisen, als die von der Forschung fokussierte höhere Bildung. Dieser nicht ganz überraschende Befund weist darauf hin, dass Akteure es als besondere Aufgabe der Volksschule ansahen, beide Geschlechter gleichermassen «auf dem Wege naturgemässer Entwicklung zum ächten Menschentuhm» zu erziehen (Dula 1873, S. 10). Dieser Anspruch findet sich in Aussagen damaliger Curriculumverantwortlichen, die wie Kettiger (1854) fanden, in der Volksschule sei «auch im Mädchen bei der Erziehung vor allem der Mensch und erst nach diesem die zukünftige Stellung in der Gesellschaft ins Auge zu fassen» (S. 39).

Das Argument für eine Kompensation und eine aufwandsmässig gleiche Beschulungszeit lautete: eine ausgleichende Gerechtigkeit herstellen. Von Lehrpersonen aus der Handarbeitsbewegung für Knaben wurde das Gerechtigkeitsargument auch bewusst eingesetzt, um eigene, standespolitische Interessen durchzusetzen und damit neue Unterrichtsinhalte $\mathrm{zu}$ implementieren. 
Die Schwierigkeit vorauszusehen, welches Wissen Schülerinnen und Schüler in Zukunft benötigten, ist ein weiterer Grund, der erklären könnte, warum Akteure trotz explizitem Bezug auf die duale Natur der Geschlechter für einheitliche Volksschulcurricula eintraten. Es sei zwar «vollkommen richtig», so Frauenbildner Schmid-Linder (1886), dass Mädchen den «Beruf einer Hausfrau» von «der Natur und den gegebenen bestehenden Verhältnissen» sowie "von Gott» zugewiesen bekämen; allerdings könne die Schule sie nicht «nur für diesen Beruf tüchtig ... machen", dessen Ausübung ja schliesslich einen nicht immer vorhandenen «Hausvater ... Ernährer und Erhalter der Familie» voraussetze (S. 4, Hervorhebung im Original).

\section{Strukturelle und finanzpolitische Faktoren}

Gemäss unseren Daten finden sich die differenziertesten Lehrpläne in städtischen Gebieten. Dies könnte auf die Prädominanz des dualen Geschlechtermodells im städtischen Bürgertum zurückgeführt werden. Eine alternative Erklärung für den Unterschied zwischen Stadt und Land offerieren Tyack und Hansot (1992): Sie gehen der Frage nach, warum sich das Modell der «identical coeducation» (S. 4) in ruralen Gebieten der USA im 19. Jahrhundert fast diskussionslos durchsetzte. Zentrale Kriterien der Schulgestaltung, so ein Ergebnis, waren die Nähe zwischen Schule und Wohnort sowie die von der Gemeinschaft für das Schulwesen aufzubringenden Kosten. Dass die Schule kostengünstig und nahe gelegen war, schien Eltern wichtiger, als dass sie ein genderspezifisches Curriculum anbot.

Nach dem Kompensationsprinzip gestaltete Differenzierungen können somit auch mit Bezug auf schulstrukturelle Rahmenbedingungen begründet werden: Auf dem Land, wo häufig das Prinzip ein Lehrer - eine Schule - eine Klasse waltete, hätte die Durchsetzung eines vollständig genderdifferenzierten Programms - das auch eine Verdoppelung der (Infra-)Strukturen bedeutet hätte $-\mathrm{zu}(\mathrm{zu})$ hohen Kosten geführt.

\section{Standespolitische Interessen}

Ein nicht zu unterschätzender Faktor sind die Interessen der in der Lehrplanarbeit involvierten Akteure. Deliberationen um neue Curricula aktivieren stets Interessengemeinschaften, deren Karriere und Status von der Etablierung oder Erhaltung bestimmter Fachbereiche oder Schultypen abhängen - hier trat vor allem die an den bildungspolitischen Rand gedrängte weibliche Lehrerschaft auf den Plan.

So war es bspw. der Schweizerische Gemeinnützige Frauenverein, der 1897 alle Kantonsregierungen dazu aufrief, obligatorische Haushaltungslektionen einzuführen und alle Schulen mit Schulküchen auszustatten (Benz, 1902). In Basel-Stadt forderten Frauenvereine 1910 vom Erziehungsdepartment «eine wöchentliche Stunde obligatorischer Unterricht in Gesundheitslehre» in der Mädchensekundarschule, die «aus Gründen des Taktes besser durch eine Lehrerin gegeben [werden sollte]» (Erziehungsdepartment BS, 1912). Erziehungsrat Fritz 
Mangold aber stellte sich explizit gegen die Einrichtung einer solchen Frauendomäne im Volksschulbereich. In den Vorarbeiten zu Schaffhauser Lehrplänen von 1928 waren es wiederum die Arbeitsschulinspektorin und der Arbeitslehrerinnenverein, die den männlich besetzten Erziehungsrat kritisierten, weil er beabsichtigte, die Handarbeitsstunden zugunsten von Geometrie zu halbieren; so würden Mädchen, «für ihre spätere Stellung als Hausfrau schwer benachteiligt» (Erziehungsrat SH, 1928).

Diese Beispiele zeigen: In der Lehrplanarbeit geht es immer auch um die Verteilung der zur Verfügung stehenden Mittel, Kompetenzen und Arbeitsplätze. Gewisse Fachbereiche zur Männer- bzw. Frauendomäne zu deklarieren, scheint in dieser Hinsicht eine durchaus gewinnbringende Strategie zu sein. Insbesondere Frauen konnten so über lange Zeit Domänen für sich beanspruchen, die ihnen sonst verwehrt wurden; aber auch Männer bedienten sich instrumentell solcher Strategien. Die kantonal und zeitlich unterschiedliche Schlagkraft geschlechtsspezifischer Interessengemeinschaften könnte also ein weiterer Erklärungsfaktor für die heterogene Ausprägung geschlechtsspezifischer Differenzierungen sein. Spätestens mit dem Gleichstellungsartikel wurden solche machtpolitischen Strategien obsolet. Der Abbau von Differenzierungen hatte mittelfristig personal- und anstellungspolitische Veränderungen, insbesondere bei der Ausbildung und Anstellung von (Fach-)Lehrerinnen zur Folge.

\section{Sozioökonomische Bedürfnisse}

Die Einführung mädchenspezifischer Fächer ab Mitte des 19. Jahrhunderts als Massnahme gegen den Pauperismus zielte auf die Lösung gesellschaftlicher Probleme in Bezug auf Ernährung und Gesundheit, die man insbesondere bei der Arbeiterschaft lokalisierte (Masoni, 2016; Manz, Giudici \& Masoni, in Druck). Auch hinter der Einführung von knabenspezifischen Schulfächern standen ähnliche volkswirtschaftliche Überlegungen. Solche Fächer nahmen daher in Schulen mit geringen Leistungsanforderungen einen prominenten Platz ein.

Auch bei den reformpädagogischen Bestrebungen nach 1900 überlagerten sich geschlechts- und leistungs- bzw. schichtbezogene Argumente: Die Aufwertung praktischer Tätigkeiten sowie an die konkrete Lebenswelt der Kinder ausgerichtete, praxisorientierte Lehrinhalte, denen Lehrplanreformer dieser Zeit einen besonderen pädagogischen Wert attribuierten, finden sich vor allem in den Schultypen mit Grundansprüchen. Mit der Ausdifferenzierung der Sekundarstufe I im Laufe des 20. Jahrhunderts und der Einbindung neuer anwendungsorientierter, manueller Unterrichtsgegenstände in die Volksschule wurde insbesondere in leistungsschwächeren Klassen eine doppelte Differenz konstruiert (Criblez \& Manz, 2015).

Unser Fazit: Curricula scheinen eher an konkrete und wechselnde Bedürfnisse in Wirtschaft und Gesellschaft orientiert, als an ein anthropologisch begründetes, über Schicht, Zeit und Kultur stehendes duales Geschlechtermodell. Zwar war die Lebens- und Berufswelt der Schülerinnen und Schüler 
lange geschlechtsspezifisch differenziert, sie unterschied sich jedoch zusätzlich auch schicht- und regionalspezifisch. Und sie unterscheidet sich darin noch heute. Trotz Koedukation, formaler Gleichheit der Lerninhalte und damit auch der Lernzeiten, bleibt es umstritten, ob daraus wirklich eine gleiche Beschulung für Mädchen und Knaben resultiert. Wie die eingangs präsentierten aktuellen Debatten zur genderspezifischen Konnotation von Schulfächern zeigt, prägt das duale, geschlechterdifferenzierte Denken bis heute die Diskussion um «richtige» Beschulung und Lernzeiten.

\section{Anmerkungen}

1 Wir verwenden in diesem Beitrag den Begriff Lernzeit als die durch Curricula regulierte Zeit, die Schülerinnen und Schüler für das Lernen schulischer Inhalte in und von der Institution Schule zur Verfügung gestellt wird. Wir verwenden den Begriff daher nicht wie in der Lehr-Lern- oder Unterrichtsforschung, um die zeitliche Dimension von Lernprozessen von Schülerinnen und Schüler oder um spezifische Unterrichtsphasen zu bezeichnen.

2 Wir orientieren uns grundsätzlich an der angelsächsischen Tradition und verwenden den Begriff Curriculum, der - im Gegensatz zu den Begriffen Lehrplan/Plan d'étude - in einem breiteren Verständnis sowohl das in normativen Dokumenten wie Schulgesetzen und Lehrplänen kodierte schulische Wissen umfasst, dessen Strukturierung und Vermittlungsweise als auch die Perspektive auf Organisationsformen der Schule. Wir gebrauchen hingegen den Terminus Lehrplan oder Lehrplanarbeit, wenn es um die konkreten Dokumente - staatlich erlassene Lehrpläne - und deren inhaltlichen Entwicklung oder Analysen geht (vgl. Horlacher \& De Vincenti, 2014).

3 Vgl. die von Cuban (1998) beschriebenen, unterschiedlichen Curriculumformen.

4 SNF-Sinergia-Projekt (CSRII1_160810, 2013-2016) der Universitäten Zürich und Genf sowie der Pädagogischen Hochschulen FHNW, Tessin und Zürich, siehe: https:// www.uzh.ch/blog/ife-hbs/forschungsprojekte/abgeschlossen/schulwissen/. Das Projekt umfasst den Zeitraum der 1830er- bis 1990er-Jahre und zehn Kantone (AG, BS, BE, FR, GE, LU, SZ, VD, TI, ZH). Erstmalig ist eine umfangreiche Quellen- und Datensammlung entstanden, die eine zeit- und regionalvergleichende Perspektive zulässt: (a) offizielle Dokumente, die Curricula regulieren (Schulgesetze, Lehrpläne, Reglemente und Weisungen, Lehrmittel); (b) bildungspolitische Quellen zu deren Entstehungsprozess, bspw. Protokolle und interne Korrespondenz der Treffen von dafür zuständigen Kommissionen oder Gremien, Rechenschaftsberichte der kantonalen Erziehungsdepartemente oder Schriften und Positionspapiere der mit der Curriculumarbeit beauftragten Individuen; sowie (c) eine systematische Sammlung relevanter Artikel in den wichtigsten überkantonalen und z.T. kantonalen pädagogischen Zeitschriften.

5 Kritisch dazu bspw. Rang (1986).

6 In den Analysen wurde erstens die Lektionenverteilung pro Kanton und Schultyp über den gesamten Untersuchungszeitraum rekonstruiert; zweitens wurden die Schulfächer zu Fächergruppen aggregiert, um sie zu verschiedenen Zeitpunkten vergleichen zu können; drittens wurden fallspezifisch Dokumente zur Lehrplanarbeit analysiert, um Akteure, Akteurkonstellationen und spezifische Legitimationen für die Curriculumentwicklung rekonstruieren zu können.

7 Doch auch die obligatorische Schulzeit differierte in manchen Kantonen je nach Geschlecht. Gemäss Benz wurden 1902 Mädchen in Freiburg, Solothurn und Thurgau ein Jahr und in Luzern und Nidwalden zwei Jahre vor ihren männlichen Mitschülern von der Schulpflicht entbunden. Zur Entwicklung der Schulpflicht und Schulstruktur in allen Schweizer Kantonen vgl. auch www.bildungsgeschichte.uzh.ch. 
8 Bspw. in Basel-Stadt oder gewissen Sekundarschultypen mit erweitertem Leistungsanspruch in der französischsprachigen Schweiz (vgl. dazu Manz, Giudici \& Masoni, in Druck Monnier, 2014).

\section{Literaturverzeichnis}

Albisetti, J. C., Goodman, J. \& Rogers, R. (Eds). (2010). Girls' secondary education in the Western World. New York, NY: Palgrave Macmillan.

Apple, M. W. (Hrsg.). The state and the politics of knowledge. New York, NY: Routledge.

Benz, E. (1902). Der Stand der Frauenbildung in der Schweiz. In H. Lange \& G. Bäumer (Hrsg.), Handbuch der Frauenbewegung (S. 207-235). Berlin: W. Moeser.

Bourdieu, P. (2002). La domination masculine. Lonrai: Éditions du Sueil.

Bundesgericht. (1986). Bundesgericht, II. Öffentlichrechtliche Abteilung, 10. Juli 1986. Gleichstellung der Geschlechter - Schulrecht - Verfassungsgerichtbarkeit. Schweizerisches Zentralblatt für Staats- und Verwaltungsrecht, 88, 147-174.

Criblez, L. \& Manz, K. (2015). "Unterricht auf werktätiger Grundlage» oder: Die Konstruktion einer doppelten curricularen Differenz durch Geschlechter- und Leistungsdifferenzierung in den Stundentafeln der Zürcher Sekundarstufe I. In C. Groppe, G. Kluchert, \& E. Matthes (Hrsg.), Bildung und Differenz. Historische Analysen zu einem aktuellen Problem (S. 203-230). Berlin: Springer.

Crotti, C. (2006). Ist der Bildungserfolg bzw. -misserfolg eine Geschlechterfrage? Zeitschrift für Pädagogik, 52, (3), 363-374.

Cuban, L. (1998). The integration of sciences into the American secondary school curriculum. Zeitschrift für Pädagogik, Beiheft 38, 89-113.

Dula, F. (1873). Ueber Geschlechtertrennung in Primarschulen und über höhere Mädchenschulen. Zürich: J. Herzog.

EDK. (1981). Grundsätze und Empfehlungen betreffend gleiche Ausbildungschancen für Mädchen und Knaben vom 30. Oktober 1981. Zugriff am 12.10.2017, verfügbar unter: www.edk.ch

Erziehungsdepartement BS. (1912). Das Erziehungsdepartement des Kantons Basel-Stadt an den E.E. Regierungsrat (Gesundheitslehre Obligatorium). StABS: ED M1.

EDK. (1993). Empfehlungen zur Gleichstellung von Frau und Mann im Bildungswesen vom 28. Oktober 1993. Zugriff am 1.9.2918, verfügbar unter: www.edk.ch.

Erziehungsrat SH. (1928). Protokolle des Erziehungsrates 1928. Staatsarchiv SH: Schule 1/31.

Giudici, A. (2018). Explaining Swiss language education policy. Dissertation, Universität Zürich.

Giudici, A. \& Grizelj, S. (2017). National unity in cultural diversity: How national and linguistic identities affected Swiss language curricula (1914-1961). Paedagogica Historica, 57, (1-2), 137-154.

Giudici, A., Extermann, B., Bürgler, B., Masoni, G., Monnier, A., Tinembart, S. \& Wrana, D. (in Druck). Wer bestimmt die schulische Wissensordnung? Zur Veränderung von Akteurskonstellationen und Wissenspolitiken. In L. Criblez, A. Giudici, R. Hofstetter, K. Manz \& B. Schneuwly (Hrsg.), Die schulische Wissensordnung im Wandel. Schulfächer, Lehrpläne, Lehrmittel . Zürich: Chronos.

Hauri, H. (1965). Koedukation an den mittleren Schulen? - Nein. Basler Schulblatt, 37, (6), $183-187$.

Hausen, K. (1976). Die Polarisierung der «Geschlechtscharaktere». Eine Spiegelung der Dissoziation von Erwerbs- und Familienleben. In W. Conze (Hrsg.), Sozialgeschichte der Familie in der Neuzeit Europas (S. 363-393). Stuttgart: Klett.

Horlacher, R. \& De Vincenti, A. (2014). From rationalist autonomy to scientific empiricism. A history of curriculum in Switzerland. In W. P. Pinar (Ed.), International handbook of curriculum research (p. 476-492). New York, NY: Routledge. 
Kellerhals, K. (2010). Der gute Schüler war auch früher ein Mädchen: Schulgesetzgebung, Fächerkanon und Geschlecht in der Volksschule des Kantons Bern 1835-1897. Bern: Haupt.

Kettiger, J. (1854). Referat über weibliche Bildung. Liestal: Lüdin \& Walser.

[LP Elementari TI 1894]. Programma particolareggiato diinsegnamento per le scuole primarie della Repubblica e Cantone del Ticino. Lugano: Traversa fu Fabrizio.

[LP Knabensek BS 1931]. Lehrziehl der Knabensekundarschule Basel. Staatsarchiv Basel-Stadt: ED L.

[LP Mädchensek BS 1932]. Lehrplan für die Mädchensekundarschule des Kantons Basel-Stadt. Staatsarchiv Basel-Stadt: ED Reg 1 420-1.

[LP Prim BE 1897]. Unterrichtsplan für die deutschen Primarschulen des Kantons Bern. Forschungsbibliothek Pädagogische Hochschule Zürich: BE HD I 1/4. Forschungsbibliothek Pädagogische Hochschule Zürich: LU HC II 1.

[LP Prim LU 1890]. Lehrplan für die Primar- und Fortbildungsschulen des Kantons Luzern.

[LP Sek LU 1895]. Lehrplan für die Sekundarschulen des Kantons Luzern. Forschungsbibliothek Pädagogische Hochschule Zürich: LU DH II 1.

[LP Volksschule ZH 1905]. Lehrplan der Volksschule des Kantons Zürich. Zürich: Erziehungsdirektion.

Lojewski, J. (2011). Geschlecht und Studienfachwahl - fachspezifischer Habitus oder geschlechtsspezifische Fachkulturen? In P. Bornkessel \& J. Asdonk (Hrsg.), Der Übergang Schule - Hochschule (S. 279-348). Wiesbaden: VS Verlag für Sozialwissenschaften.

Mantovani Vögeli, L. (1994). Fremdbestimmt zur Eigenständigkeit. Mädchenbildung gestern und heute. Chur: Rüegger.

Manz, K., Giudici, A. \& Masoni, G. (in Druck). Hat schulisches Wissen ein Geschlecht? Der Einfluss der Strukturkategorie «Geschlecht» auf Lehrpläne und Lehrmittel. In L. Criblez, R. Hofstetter, K. Manz, B. Schneuwly \& A. Giudici (Hrsg.), Die schulische Wissensordnung im Wandel. Schulfächer Lehrpläne, Lehrmittel. Zürich: Chronos.

Masoni, G. (2016). La Cattedra ambulante di Agricoltura e i Corsi itineranti di Economia domestica: proposte formative tipiche di un territorio alpino? Percorsi di ricerca, (8), $25-36$.

Mayer, C. (1999). Bildungsentwürfe und die Konstruktion der Geschlechterverhältnisse zu Beginn der Moderne. In B. L. Behm, G. Heinrich \& H. Tiedemann (Hrsg.), Das Geschlecht der Bildung - Die Bildung der Geschlechter (S. 13-29). Berlin: Springer.

Mead, S. (2006). The truth about boys and girls. Washington, DC: Education Sector.

Monnier, A. (2014). La question de la mixité dans l'enseignement public genevois. Entre séparation, porosité et convergence (1836-1969). Histoire de l'éducation, (3), 201-219.

Rang, B. (1986). Zur Geschichte des dualistischen Denkens über Mann und Frau. In J. Dalhoff, U. Frey \& I. Schöll (Hrsg.), Frauenmacht in der Geschichte (S. 194-204). Düsseldorf: Schwann.

Schmid, P. (1986). Das Allgemeine, die Bildung und das Weib. In H.-E. Tenorth (Hrsg.), Allgemeine Bildung: Analysen zu ihrer Wirklichkeit, Versuche über ihre Zukunft (S. 202-214). Weinheim \& München: Juventa.

Schmid-Linder, C. (1886). Ueber die Einführung von Frauenarbeitsschulen. Zürich: J. Herzog.

Smith, B. G. (1981). Ladies of the leisure class. The bourgeoises of Northern France in the ninteenth century. New York, NY: Routledge.

Sullivan, A., Joshi, H. \& Leonard, D. (2010). Single-sex schooling and academic attainment at school and through the lifecourse. American Educational Research Journal, 47, (1), 6-36.

Tolley, K. (2003). The science education of American girls: A historical perspective. New York, NY: Routledge Falmer.

Tyack, D. \& Hansot, E. (1992). Learning together. A history of coeducation in American public schools. New York, NY: Russel Sage.

[VSG AG 1865]. Schul-Gesetz für den Kanton Aargau. Vom 1. Brachmonat 1865. Aarau: s.n. 
[VSG ZH 1859]. Gesetz über das gesammte Unterrichtswesen des Kantons Zürich vom 23. Dezember 1859. Officielle Sammlung der seit Annahme der Verfassung vom Jahre 1831 erlassenen Gesetze, Beschlüsse und Verordnungen des Eidgenössischen Standes Zürich, 12(1859), 243-362.

Wagner, J. R. (1870). Ueber Mädchenerziehung und Mädchenbildung. Lichtensteig: Buchdruckerei von Dr. A Stegner.

Warnende Stimme. (1842). Mittheilungen über die in den meisten Erziehungsanstalten der französischen Schweiz herrschenden pädagogischen Fehlgriffe \& Mängel. Baden: Zehnder \& Tuchschmied.

Westbury, I., Aspfors, J., Fries, A.-V., Hansén, S.-E., Ohlhaver, F., Rosemund, M. \& Sivesind, K. (2016). Organizing curriculum change: an introduction. Journal of Curriculum Studies, 48, (6), 729-743.

Willems, K. (2007). Schulische Fachkulturen und Geschlecht. Bielefeld: transcript Verlag.

Schlagworte: Curriculum, Geschlecht, Lehrplan, Koedukation

\section{Temps de l'éducation des garçons - temps de l'éducation des filles: égaux, de plus en plus égaux, inégaux? Les différenciations de genres dans le savoir scolaire}

\section{Résumé}

Les curricula et les temps de l'éducation des écoliers et des écolières ont longtemps été différents. Ce phénomène était jusqu'ici relié à une vision duelle du genre. Politiciens et pédagogues diversifiaient ces curricula car ils considéraient que les dispositions des hommes et des femmes étaient différentes et que l'école devait contribuer à leur développement. Notre analyse des programmes scolaires suisses depuis 1830 ne montre cependant pas une structure clairement duelle du savoir scolaire. Ceci prouve que les normes genres, apparemment stables, indépendantes des classes, des régions ou des époques, ne suffisent pas à expliquer ces différentiations du savoir scolaire. En effet, les curricula doivent aussi répondre aux nécessités spécifiques de certains contextes régionaux, économiques, institutionnels et de pédagogie pratique.

Mots-clés: Curriculum, genre, savoir scolaire, coéducation 


\section{Tempi d'apprendimento maschili - tempi d'apprendimento femminili: uguali, sempre più uguali o disuguali? Differenziazioni di genere del sapere scolastico}

\section{Riassunto}

Per lungo tempo, i curricoli e i tempi d'apprendimnto degli scolari e delle scolare hanno differito. Finora tale fenomeno è stato spiegato attraverso una visione duale del genere: poiché politici e pedagoghi supponevano che le disposizioni della donna e dell'uomo divergessero e che la scuola dovesse perfezionarle, diversificarono i curricoli per genere. Ma secondo la nostra analisi dei piani di studio svizzeri effettuata a partire dal 1830, appare che il sapere scolastico non era strutturato in maniera chiaramente duale. Ciò dimostra che le norme duali di genere, apparentemente stabili, indipendenti da classe, regione o tempo non bastano a spiegare tali differenziazioni del sapere scolastico. Infatti, i curricoli devono sempre soddisfare anche i bisogni specifici di determinati contesti regionali, economici, istituzionali di pronto uso pedagogico.

Parole chiave: Curriculo, genere, sapere scolastico, coeducazione

\section{Male learning times - female learning times: equal, more equal, unequal? Gender-specific differentiations of school knowledge}

\section{Summary}

For a long time, boys' and girls' curricula and learning times differed. So far, this phenomenon has been linked to norms about the duality of gender: since politicians and pedagogues assumed that men's and women's dispositions differed, and that school should perfect them, they designed differentiated curricula. However, according to our analysis, curriculum documents for Swiss primary schools since 1830 do not present such a dualistic engendered structure. Therefore, the allegedly stable, class- and timeless, and non-regional norms about the dual nature of gender cannot fully explain gender-specific differentiations of school knowledge. In fact, curricula also have to fulfil needs that are very specific to regional, economic, institutional, and pedagogic-practical contexts.

Keywords: Curriculum, gender, school knowledge, coeducation 